\title{
Hydatid cyst of the intramyocardial heart
}

\author{
A Hadi*, A Laalou, S Elkarimi and M Elhattaoui \\ Department of Cardiology-University Hospital of Mohamed VI-Marrakech-Morocco
}

\begin{abstract}
Introduction: The hydatid cyst of the heart is rare. It is a characterized by a large clinical and paraclinical polymorphism. Our objective is to illustrate through this observation the interest of screening for the cardiac localization of this affection in all patients suspected or suffering from hydatidosis.

Observation: We report the clinical case of a 47-year-old patient operated 11 years ago for two rights and left pulmonary Hydatid cysts presenting for chronic left upper abdominal and lower thoracic pain accentuated $24 \mathrm{~h}$ before his admission and in whom the clinical examination is without particularity. CT-scan shows mass $4.9 \times 5.2 \times 6.2 \mathrm{~cm}$ in inter-cardio-diaphragmatic with a thick, irregular wall reaching the left lung and contacting the lower surface of the LV and the diaphragm with loss of the interface separation with presence of calcifications. Echocardiography confirmed the presence of a heterogeneous solido-cystic mass adherent to the inferior surface of the left ventricular with presence of calcifications. The evolution was marked by the emptying of the pre-existing left cardio-phrenic cystic formation with disappearance of the cystic component and persistence of calcification peripheral to the control scan performed 2 months later making the MRI analysis difficult. The hydatid serology is Negative outcome and the diagnosis of reshaped cardiac hydatid cyst was retained given the patient's history, the evolution of the lesions and the therapeutic abstention under strict supervision was indicated.
\end{abstract}

Conclusion: This observation illustrates the interest of the systematic screening of the heart localization in any patient with or suspected of hydatidosis.

\section{Introduction}

The hydatid cyst, still called Echinococcosus, is a cosmopolit ananthropozoonosis due to the presence and development of the dog's taenia larva's form, type Echinococcosus granulosus. It can affect organs, visceras and tissues.The cardiac location is rare.This affection is characterized by a wide clinical and paraclinical polymorphism. Cardiac hydatidosis, is a severe condition which could potentially threaten prognosis due to its localisation in vital organs, and also to the cyst evolution leading to multiple local and systemic complications. The following clinical observation shows the interest of a cardiac Hydatid cyst screening, concerning a patient with extra cardiac localisation. Along with their early diagnosis and clinical care to anticipate and avoid any unfortunate complication.

\section{Observation}

A 47 years old Moroccan patient, living in rural area, having frequent contact with dogs, has been operated 18 years ago on a right Hydatid pulmonary cyst removal, then 5 years after on a left Hydatid pulmonary cyst, with smooth postoperative recoveries. Symptoms begun 3 months earlier, with recurrent left upper abdominal and lower thoracic pain, increased 24 hours before his admission, without hemoptysia or sputum or dyspnea, without weight loss, fiever or general state deterioration. Clinical examination was unremarkable, electrokardiogram and abdominal echographia were normal.

The face thoracic radiography visualized a cardiomegaly with an alvéolo-interstitial left basithoracic opacity and an attraction of the elements of the médiastinum towards the left lung's hemifield (Figure $1)$.

The thoracic Angioscanner objectified a badly limited cystic formation left inter-cardio-diaphragmatic of $4.9 \times 5.2 \times 6.2 \mathrm{~cm}$ with thick and irregular wall presenting calcifications strongly suggestive of a

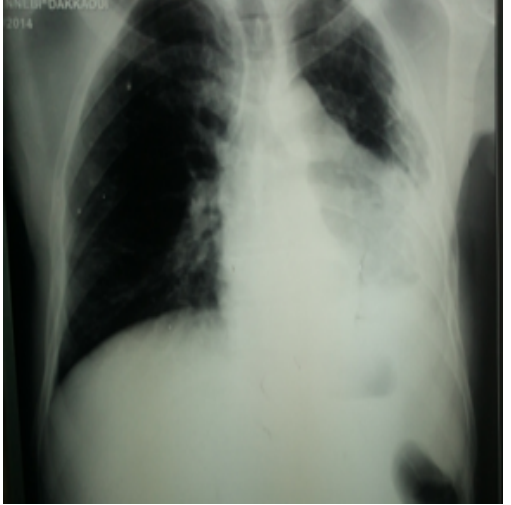

Figure 1. Chest X-ray : Cardiomegaly with left basithoracic opacity

Hydatid cyst arriving at the contact of the left lung and at the contact of the lower face of the left ventricular and the diaphragm with loss of the interface of separation with a left after effected lung responsible for an attraction of mediastinum's elements and the controlateral lung and presence of a condensation's site of left basithoracic pulmonary parenshyma ( Figure 2 ).The echocardiography showed a heterogeneous solido-cystic formation with presence of calcifications of $60 \mathrm{~mm} /$ $40 \mathrm{~mm}$. This formation is very adherent to the lower and the posterolateral wall of the left ventricular which repulses it without compressing it. (Figure 3-5). However, the hydatid serology was negative and a

*Correspondence to: A Hadi, Department of Cardiology-University Hospital of Mohamed VI-Marrakech-Morocco; Tel: +212641524330; E-mail: abdelalizate@hotmail.fr

Received: December 03, 2018; Accepted: December 10, 2018; Published: December 13, 2018 


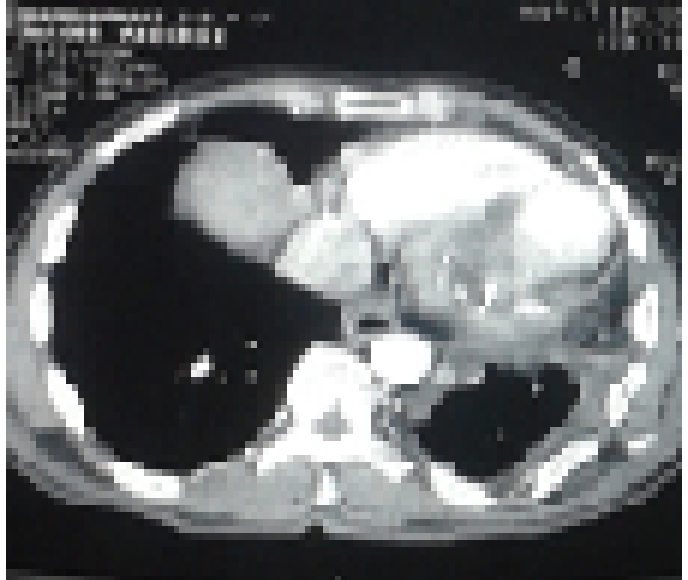

Figure 2. bis: CT scan in axial section: large cystic formation with peripheral calcification adhered to the posteriorwall of the left ventricular

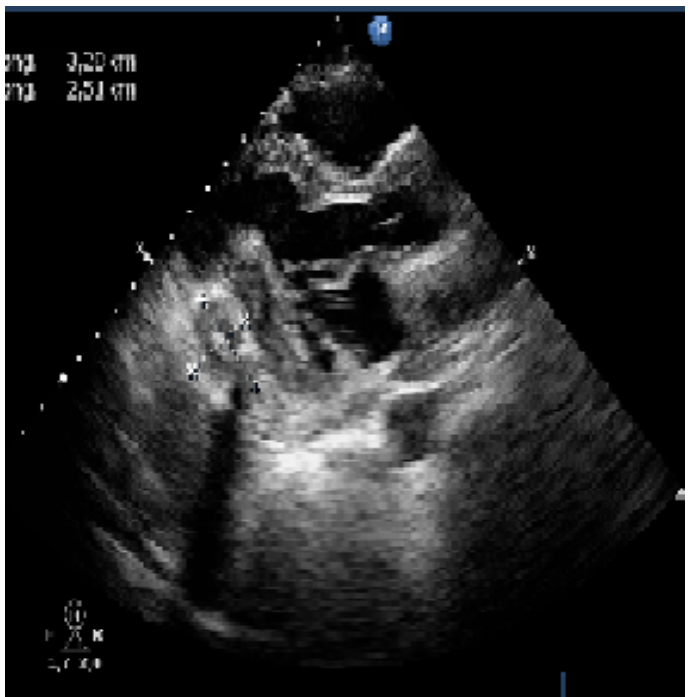

Figure 3. 2D echocardiography-parasterna long axis incidence: Heterogeneous solidocystic formation very adherent to the inferolateral wall of the left ventricular

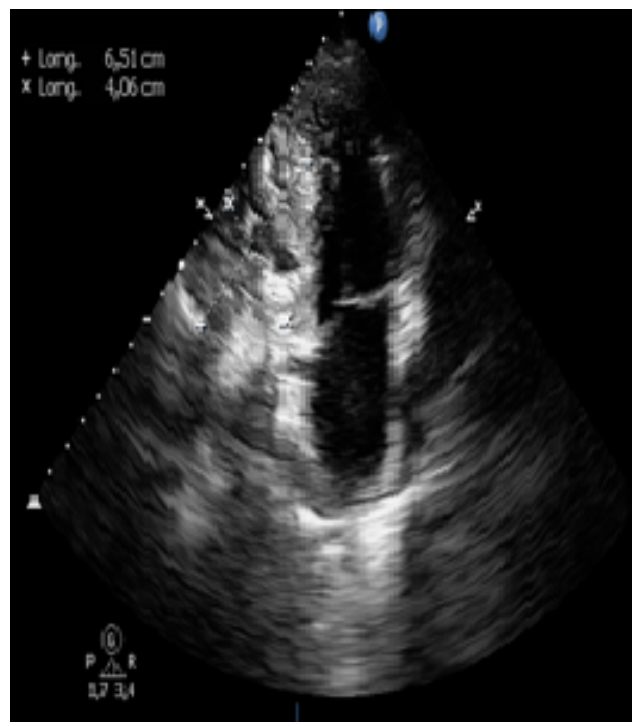

Figure 4. 2D Echocardiography-apical 2 cavity incidence: Heterogeneous solidocystic formation very adherent to the inferolateral wall of the left ventricular

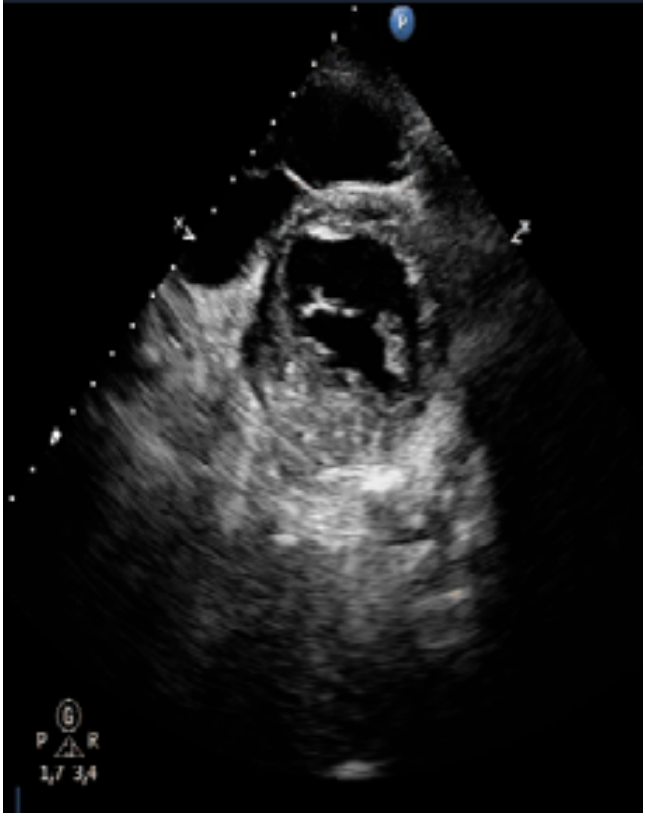

Figure 5. 2D Echocardiography- parasternale short axisincidence: Heterogeneous solidocystic formation very adherent to the inferolateral wall of the left ventricular

cardiac MRI was indicated, but its results were judged obsolete because the cystic component disappeared, giving way to many calcifications. Therefore, a second morphogical examination was needed 6 months later, for the patient was clearly still asymptomatic. The transthoracic Echocardiogram performed on this patient came out with the same results showed above, while the CT Angiography showed the voidance of the cystic formation that once occupied the former left cardiophrenic area, and whose remains measure $4.9 \times 1.9 \times 6.2 \mathrm{~cm}$. In the other hand, the cystic component completely disappeared, with air bubbles trapped inside of what is left of it, probably due to a premature rupture leading to calcifications appearing there, too. This is probably linked to a cardiac cyst that has been ruptured, then calcified.

Most of the time, this formation is close to the left liver lobe, the left ventricular and to the Lung's Inferior lobe (Figure 6). Nonetheless, the limits or even the expansion of the lesions were hardly studied because of the calcifications, for they were responsible of a bad transmission of the acoustic voids, in addition to amajor acoustic shadowing.The Hydatid serology made as a subsequent check-up remained negative. Therefore, the diagnosis that seemed adequate was a "Ruptured, then inactive Cardiac Hydatid Cyst."

Since this patient's lesions were stable and inactive, and no symptoms were ever found, a therapeutic abstention has been decided, along with regular check-ups, both biological and morphological.

After three years from the beginning of the check-ups, the patient is still asymptomatic, with a well-preserved general condition.

\section{Discussion}

The cardio - pericardial hydatidosis is rare $(0,5$ to $2 \%$ of all the Hydatid localizations and $2,6 \%$ of the intrathoracic Hydatid localizations) and comes essentially from the coronary circulation after crossing of the hepatic and lung filter. The localization of the cyst at the left heart is 3 times more common than at the straight heart: 64 to $89 \%$ for the left cavities again stonly 15 to $31 \%$ for the straight cavities because of the rich vascularisation of the wall of the left cavities [1]. The 


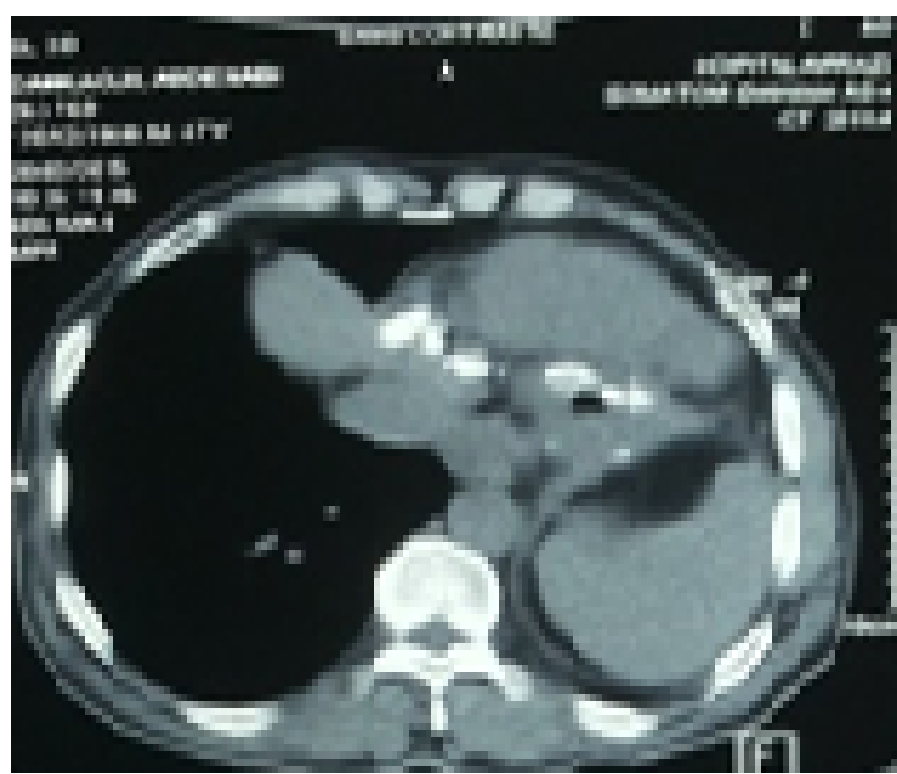

Figure 6. CT scan in axial section : disappearance of cystic formation with persistent peripheral calcification adhered to the posterior wall of the left ventricular

development of the Hydatid cyst in the heart is usually subepicardial for the left heart localizations and sub -endocardal for those of the straight heart, considering the regime of low pressure of the right cavities. This explains the possibility of intrapericardial break in the left localizations and intracavitial in the straight damages. The very slow growth of the cyst within the myocardium explains the delayed diagnosis which is often made towards the 3rd decade. Approximately 60 to $65 \%$ of hydatid cardiac cysts are isolated and 35 to $40 \%$ are associated to essentially hepatic and lung visceral localizations [1,2].The cardiac hydatidosis is characterized by a biganatomo-clinical polymorphism, the clinical signs are very variable and depend directly on the size and on the localization of Hydatid cysts, what explains the difficulty of the diagnosis at the clinical phase [3]. The thoracic radiography face and profile can detect a cardiomegaly with a round or oval image, with an hydric tone, homogeneous, deforming the outlines of the heart and the calcifications can be present bounding the outlines of this mass which can take the aspect of calcified inhomogenous plates or giving to the mass the aspect of an eggshell. The two-dimensional transthoracic echocardiography (ETT) is the key examination for the diagnosis of the cardiac hydatidosis; she shows a fluid constitution rounded ,homogeneous or containing small vesicles. The ETT or better the trans-esophageal ultrasound (ETO) allows to locate, to count and to specify the limits of the Hydatid cyst of the heart. She also provides the detection of complications like cracking or break and the impact on the cardiac function. Contrary to the fluid typical aspect, certain cysts have a solid aspect in the ultrasound causing the problem of differential diagnosis with the primitive or secondary tumors of the heart. This aspect is explicable by degenerative or infectious phenomena. The scanner multicups allows a morphological and extension sheet thanks to its spatial resolution and thanks to the possibilities of muliplanes reconstructions. On the other hand, the MRI can bring interesting information in case of uncertainty of diagnosis, or in case of conflict between the echocardiography and the tomodensitometry. She shows the cyst in hyposignal T1 and in hypersignal T2 $[3,4]$. The serological diagnosis of the hydatidosis is realized by various techniques: ELISA, hémagglutination, IEF [5], Immunoblot (presence of bands). It is necessary to couple two techniques, the one qualitative (ELISA), the other one quantitative (IB). But the serological reactions should be interpreted with caution. If they are positive, with a significant rate, the diagnosis is held; if they are negative, we cannot conclude. The IB allows to raise the diagnosis with a delicate interpretation. The interest of the serology is to give the notion of viable cyst or inactive cyst: in practice, a cystis called inactive when it is mummified, calcified, $<5$ $\mathrm{cm}$, by fortuitous discovery, with negative serology. The serological reactions permit to control the therapeutic efficiency: disappearance after treatment in 12 in 18 months; if ré-ascent, you must fear of a second recurrence or a reinfection [3].

The treatment is surgical because the spontaneous evolution is mortal in more or less brief term. The surgical treatment contains a cystectomy or at least a draining of the cyst which is sterilized by the intracystic injection of a parasiticidal solution (hypertonicsalty solution). A treatment with fluoromébendazole or albendazole $15 \mathrm{mg}$ $/ \mathrm{kg} / \mathrm{j}$ in two taken (cure of 4 weeks spaced out of 15 days during 6 à 12 mounths to see more) associated was recommended [3,4]. After the surgical operation, a clinical, electrocardiographical, biological, radiological and ultra sound echographical follow up is imperative for the seek of a possible recurrence. [5-8]

\section{Conclusion}

Hydatidosis affecting the heart is rare. She is burdened with potentially serious complications. The severity of this localization necessitates its systematic screening echocardiographic in any patient suffering from or suspected of hydatidosis and its prevention is the best way to reduce the frequency of this condition, especially in endemic countries.

\section{References}

1. Elkouby A, Vaillant A, Comet B, Malmejac C, Houel J (1990) Cardiac hydatidosis Review of recent literature and report of 15 cases. Ann Chir 44: 603-610. [Crossref]

2. Trigano JA, Mourot F, Talmoudi T, Malmejac C, Torresani J, et al. (1985) Symptomatology of hydatid cyst of the heart. Study of a continuous series of 13 cases and value of x-ray computed tomography. Arch Mal Coeur Vaiss 78: 1895-1899. [Crossref]

3. Jouhadi Z, Ailal F, Dreoua N, Eddine AZ, Abid A, et al. (2004) [Cardiac hydatid cyst. Two cases in children]. Med 33 Press: 1260-1263. [Crossref]

4. Endale Tefera, Joseph Knapp, Michael Teodori (2003) Hydatid cyst of the interventricular septum of the heart: aspect in multibarrette scanner and in MRI. $J$ radiol 84: 614-616. [Crossref]

5. Bréchignac X, Durieu I, Perinetti M, Gerinière L, Richalet C, et al. (1997) Hydatid cyst of the heart. Med Press 26: 663-665. [Crossref]

6. Rosenberg T, Panayiotopoulos YP, Bastounis E, Palambros E, Balos P (1993) Acute abdominal aorta embolism caused by primary cardiac echinococcus cyst. Eur J Vasc Surg 7: 582-585. [Crossref]

7. Thameur H, Chenik S, Abdelmoulah S, M Bey, Hachicha S, et al. (2000) Thoracic hydatidosis. A review of 1619 boxes. Rev Pneumol Clin 56: 7-15. [Crossref]

8. Bashour TT, Alali AR, Mason DT, Saalouke M (1996) Echinococcosis of the heart clinical and echocardiographic features in 19 patients. Am Heart $J$ 132: 1028-1030. [Crossref]

Copyright: (C2018 Hadi A. This is an open-access article distributed under the terms of the Creative Commons Attribution License, which permits unrestricted use, distribution, and reproduction in any medium, provided the original author and source are credited. 